\title{
Healthcare-associated infections and antimicrobial resistance in Canadian acute care hospitals, 2014-2018
}

\author{
Canadian Nosocomial Infection Surveillance Program ${ }^{1}$
}

\begin{abstract}
Background: Healthcare-associated infections (HAls) and antimicrobial resistance (AMR) pose serious threats to the health of Canadians due to increased morbidity, mortality and healthcare costs. Epidemiologic and laboratory surveillance data, collected through the Canadian Nosocomial Infection Surveillance Program, are used to inform infection prevention and control and antimicrobial stewardship programs and policies. The objective of this study was to describe the epidemiologic and laboratory characteristics and trends of HAls and AMR from 2014 to 2018 using surveillance data provided by Canadian hospitals participating in the Canadian Nosocomial Infection Surveillance Program.
\end{abstract}

Methods: Data were collected from 70 Canadian sentinel hospitals between January 1, 2014 and December 31, 2018 for Clostridioides difficile infection (CDI), methicillin-resistant Staphylococcus aureus bloodstream infections, vancomycin-resistant Enterococci bloodstream infections and carbapenemase-producing Enterobacteriaceae. Case counts, rates, outcome data, molecular characterization and antimicrobial resistance profiles are presented.

Additionally, hospital-level Escherichia coli antibiogram data were collected and are described.

Results: Increases in rates per 10,000 patient-days were observed for methicillin-resistant $S$. aureus bloodstream infections $(59 \% ; 0.66-1.05, p=0.023)$ and vancomycin-resistant Enterococci bloodstream infections (143\%; $0.14-0.34, p=0.023)$. However, CDI rates decreased by $12.5 \%$ between 2015 and 2018 (from 6.16-5.39, p=0.042). Carbapenemase-producing Enterobacteriaceae infection rates remained low and stable whereas colonization increased by $375 \%(0.04-0.19 ; p=0.014)$.

Conclusion: Ongoing efforts to prevent HAls and reduce AMR in Canada require consistent, standardized surveillance data from acute care hospitals. Increased collaboration with provincial, territorial and international partners in infection prevention and control, as well as antimicrobial stewardship, will be essential in reducing the burden of observed HAls (including antimicrobial resistant organisms).

Suggested citation: Canadian Nosocomial Infection Surveillance Program. Healthcare-associated infections and antimicrobial resistance in Canadian acute care hospitals, 2014-2018. Can Commun Dis Rep 2020;46(5):99-112. https://doi.org/10.14745/ccdr.v46i05a01

Keywords: healthcare-associated infections, community-associated infections, antimicrobial resistance, surveillance, Clostridioides difficile infection, methicillin-resistant Staphylococcus aureus, vancomycin-resistant Enterococci, carbapenemase-producing Enterobacteriaceae, antibiogram, Escherichia coli, Canadian Nosocomial Infection Surveillance Program

\section{Introduction}

Healthcare-associated infections (HAls) including antimicrobial resistant organisms (AROs) pose a serious risk to the safety and quality of care delivered to patients globally, including in Canada. HAls cause significant morbidity and mortality among patients and result in increased healthcare costs (1-4). A 2017 point prevalence survey among participating Canadian hospitals estimated that $7.9 \%$ of patients had at least one $\mathrm{HAl}$; results that are similar to those from a 2016-2017 study by the European 
Centre for Disease Prevention and Control that estimated HAI prevalence among tertiary care hospitals to be $7.1 \%(5,6)$. A study conducted in the European Union and European Economic Area in 2015 estimated that 2,609,911 new cases of HAl occur every year, corresponding to an annual burden of 501 disability-adjusted life years per 100,000 general population (7).

Antimicrobial resistance (AMR) is a growing healthcare concern, with increased resistance levels detected in humans worldwide (8). Antimicrobial resistant infections cause at least 50,000 deaths each year across Europe and the United States (US) alone (9). Close monitoring of AMR is vital for detecting and responding to emerging trends and patterns of resistance and thus to effectively controlling and treating HAls.

In Canada, the Public Health Agency of Canada (PHAC) collects national data on various HAls and AMR through the Canadian Nosocomial Infection Surveillance Program (CNISP). This program was established in 1995 as a partnership between PHAC, the Association of Medical Microbiology and Infectious Disease Canada and sentinel hospitals across Canada. The goal of CNISP is to help facilitate the prevention, control and reduction of HAls and AROs in Canadian acute care hospitals through active surveillance and reporting.

Reflecting the core components of infection prevention and control of the World Health Organizations (10), CNISP performs consistent, uniform surveillance to reliably measure HAI burden, establish benchmark rates for internal and external comparison, identify potential risk factors and allow for the assessment of specific interventions to improve the quality of patient care. Data provided by CNISP directly supports the goals outlined in the 2017 Pan-Canadian Framework for Action for tackling antimicrobial resistance and antimicrobial use (11).

In this report, we describe the most recent $\mathrm{HAl}$ and AMR surveillance data collected from CNISP participating hospitals between 2014 and 2018.

\section{Methods}

\section{Design}

Canadian Nosocomial Infection Surveillance Program conducts prospective, sentinel surveillance for HAls (including AROs) and collects annual hospital-level antibiograms.

\section{Case definitions}

Standardized case definitions for healthcare-associated (HA) and community-associated (CA) infections were used. Refer to Appendix A for full case definitions.

\section{Data sources}

Epidemiologic data: Between January 1, 2014 and December 31, 2018, participating hospitals submitted epidemiologic data on cases meeting the respective case definitions for Clostridioides difficile infection (CDI), methicillin-resistant Staphylococcus aureus bloodstream infections (MRSA BSI), vancomycin-resistant Enterococci bloodstream infections (VRE BSI) and carbapenemase-producing Enterobacteriaceae (CPE) infections and colonizations. Community-associated CDI surveillance was launched in 2015 and CA-CDI cases have been included since then. In 2018, 70 hospitals across Canada participated in HAl surveillance and are further described in Table 1.

\section{Table 1: Summary of hospitals participating in the Canadian Nosocomial Infection Surveillance Program, by region, 2018}

\begin{tabular}{|c|c|c|c|c|}
\hline $\begin{array}{c}\text { Details of } \\
\text { participating } \\
\text { hospitals }\end{array}$ & Western ${ }^{a}$ & Central $^{b}$ & Eastern ${ }^{c}$ & Total \\
\hline $\begin{array}{l}\text { Total number of } \\
\text { hospitals }\end{array}$ & 26 & 28 & 16 & 70 \\
\hline \multicolumn{5}{|l|}{ Hospital type } \\
\hline Adult ${ }^{d}$ & 11 & 18 & 8 & 37 \\
\hline Mixed & 12 & 6 & 7 & 25 \\
\hline Pediatric & 3 & 4 & 1 & 8 \\
\hline \multicolumn{5}{|l|}{ Hospital size } \\
\hline $\begin{array}{l}\text { Small } \\
\text { (1-200 beds) }\end{array}$ & 7 & 6 & 8 & 21 \\
\hline $\begin{array}{l}\text { Medium } \\
\text { (201-499 beds) }\end{array}$ & 13 & 15 & 8 & 36 \\
\hline $\begin{array}{l}\text { Large } \\
\text { (500+ beds) }\end{array}$ & 6 & 7 & 0 & 13 \\
\hline \multicolumn{5}{|c|}{ Admissions and discharge } \\
\hline $\begin{array}{l}\text { Total number of } \\
\text { beds }\end{array}$ & 9,277 & 10,354 & 3,038 & 22,669 \\
\hline $\begin{array}{l}\text { Total number of } \\
\text { admissions }\end{array}$ & 440,400 & 485,416 & 103,519 & $1,029,335$ \\
\hline $\begin{array}{l}\text { Total number of } \\
\text { patient days }\end{array}$ & $3,217,499$ & $3,521,438$ & 926,355 & $7,665,292$ \\
\hline
\end{tabular}

Participating hospitals submitted epidemiologic (demographic, clinical and outcome data) and denominator data (associated patient-days and patient-admissions) electronically through the Canadian Network for Public Health Intelligence platform; a secure on-line data entry system. Standardized protocols and case definitions were reviewed annually by expert working groups and annual training sessions were provided for data submission. Data quality within CNISP projects has been evaluated periodically $(12,13)$.

Laboratory data: Patient-linked laboratory isolates were sent to the PHAC's National Microbiology Laboratory (NML) for molecular characterization and susceptibility testing. MRSA BSI, VRE BSI, CPE and pediatric CDI isolates were submitted year round. Adult $\mathrm{CDI}$ isolates were submitted during a targeted two-month period from March 1 to April 30 each year. 
Antibiogram data: Hospitals submitted annual hospital-level antibiogram data on all inpatient and outpatient clinical Escherichia coli isolates (including blood, urine and other clinical isolates such as respiratory, skin, soft tissue and surgical sites). Duplicate isolates were removed as per Clinical and Laboratory Standards Institute guidelines (14). As of 2018, there was no minimum number of isolates required for hospital reporting (prior to 2018, the minimum cut off for reporting was 30 isolates/ hospital).

Statistical analysis: The HAl rates were calculated and represent infections and/or colonizations identified in patients admitted (inpatients) to CNISP-participating hospitals and calculated by dividing the total number of cases by the total number of patient admissions (multiplied by 1,000) or patient-days (multiplied by 10,000 ). The HAl rates were reported nationally and by region (Western: British Columbia, Alberta, Saskatchewan and Manitoba; Central: Ontario and Quebec; Eastern: Nova Scotia, New Brunswick, Prince Edward Island and Newfoundland and Labrador). The territories did not submit data to PHAC. The Mann-Kendall test was used to test trends over time. Significance testing was two-tailed and differences were considered to be significant at $p$-value $\leq 0.05$.

Where available, outcome data were reported for HAls using attributable and all-cause mortality. Attributable mortality was defined as the number of deaths per $100 \mathrm{HAl}$ cases where the $\mathrm{HAl}$ was the direct cause of death or contributed to death 30 days after the date of the first positive laboratory or histopathology specimen. All-cause mortality was defined as the number of deaths per $100 \mathrm{HAl}$ cases 30 days following positive culture.

\section{Results}

\section{Clostridioides difficile infection}

Between 2015 and 2018, the incidence of CDI decreased from 6.16 to 5.39 infections per 10,000 patient-days ( $p=0.042$ )

(Table 2). A decreasing trend was observed in HA-CDI rates $(-14.9 \%, p=0.042)$ and CA-CDI rates $(-12.3 \%, p=0.174)$

(Table S1.1). Regionally, HA-CDI rates have decreased across all regions except in the East. For CA-CDI, Eastern and Central region rates have decreased between 2015 and 2018 while Western rates have remained the same. Adult hospitals have consistently had higher rates of $\mathrm{HA}$ and CA-CDI compared to mixed and pediatric hospitals. Attributable mortality decreased from 3.0 to 1.3 deaths per 100 cases from 2015 to 2018 .

Antimicrobial resistance to moxifloxacin among CDI isolates decreased by $13.7 \%$ between 2015 and 2018, with no significant differences between HA and CA-CDI (Table S1.2). While all tested $C$. difficile strains were susceptible to vancomycin, there was a single case of metronidazole resistance in 2018. From 2015
Table 2: Clostridioides difficile infection data, Canada, 2015-2018

\begin{tabular}{|c|c|c|c|c|c|c|c|c|}
\hline \multirow{2}{*}{$\begin{array}{c}\text { C. difficile } \\
\text { infection data }\end{array}$} & \multicolumn{8}{|c|}{ Year } \\
\hline & \multicolumn{2}{|c|}{2015} & \multicolumn{2}{|c|}{2016} & \multicolumn{2}{|c|}{2017} & \multicolumn{2}{|c|}{2018} \\
\hline \multicolumn{9}{|c|}{ Number of infections and incidence rates } \\
\hline $\begin{array}{l}\text { Number of } C \text {. difficile } \\
\text { infection cases }\end{array}$ & & 4,170 & & 4,008 & & 4,012 & & 3,843 \\
\hline $\begin{array}{l}\text { Rate per } 1,000 \text { patient } \\
\text { admissions }\end{array}$ & & 4.62 & & 4.34 & & 4.28 & & 4.07 \\
\hline $\begin{array}{l}\text { Rate per } 10,000 \\
\text { patient-days }\end{array}$ & & 6.16 & & 5.77 & & 5.67 & & 5.39 \\
\hline $\begin{array}{l}\text { Number of reporting } \\
\text { hospitals }\end{array}$ & & 66 & & 67 & & 68 & & 68 \\
\hline $\begin{array}{l}\text { Attributable mortality } \\
\text { rate per } 100 \text { cases }(\%)^{\mathrm{b}}\end{array}$ & & 3.0 & & 2.4 & & 2.3 & & 1.3 \\
\hline $\begin{array}{l}\text { Antimicrobial } \\
\text { resistance }^{c}\end{array}$ & $\mathrm{~N}$ & $\%$ & $\mathrm{~N}$ & $\%$ & $\mathrm{~N}$ & $\%$ & $\mathrm{~N}$ & $\%$ \\
\hline Clindamycin & 194 & 26.0 & 145 & 22.1 & 149 & 22.0 & 307 & 48.7 \\
\hline Moxifloxacin & 185 & 24.8 & 103 & 15.7 & 114 & 16.9 & 70 & 11.1 \\
\hline Rifampin & 14 & 1.9 & 9 & 1.4 & 14 & 2.1 & 10 & 1.6 \\
\hline Metronidazole & 0 & 0.0 & 0 & 0.0 & 0 & 0.0 & 1 & 0.2 \\
\hline $\begin{array}{l}\text { Total number of } \\
\text { isolates tested }\end{array}$ & 745 & N/A & 657 & N/A & 676 & N/A & 631 & N/A \\
\hline
\end{tabular}

Abbreviations: $C$. difficile, Clostridioides difficile; N/A, not applicable

a All C. difficile strains from 2015 to 2018 submitted to National Microbiology Laboratory were susceptible to tigecycline and vancomycin

Deaths where C. difficile infection was the direct cause of death or contributed to death 30 days after the date of the first positive lab specimen or positive histopathology specimen. Mortality data are collected during the two-month period (March and April of each year) for adults (age 18 years and older) and year-round for children (age one year to less than 18 years old). Among pediatric patients, there was no death attributable to healthcare-associated $C$. difficile infection c $C$. difficile infection isolates are collected for resistance testing during the two-month period (March and April of each year) for adults (age 18 years and older) and year-round for children (age one year to less than 18 years old) from admitted patients only

d Total number reflects the number of isolates tested for each of the antibiotics listed above

to 2018 , the proportion of ribotype 027 associated with NAP1 decreased for both $\mathrm{HA}$ and $\mathrm{CA}-\mathrm{CDI}$, though the decrease was more prevalent among HA-CDI cases (Table S1.3).

\section{Methicillin-resistant Staphylococcus aureus bloodstream infections}

Between 2014 and 2018, overall MRSA BSI rates increased by $59.1 \%$ (0.66 to 1.05 infections per 10,000 patient days, $p=0.023$ ) (Table 3). An increasing trend in incidence was observed for CA-MRSA BSI (150\%, $p=0.05)$ and HA-MRSA BSI $(27.5 \%, p=0.05)$ (Table S2.1). In 2018, HA and CA-MRSA BSI rates were highest in Western Canada (0.57 and 0.64 infections per 10,000 patient days, respectively). Among hospital types, HA and CA-MRSA BSI rates remained highest in mixed hospitals compared with adult and pediatric hospitals. All-cause mortality fluctuated from 2014 to 2018 ; ranging from $16.4 \%$ (2017) to $24.9 \%$ (2014) (Table 3 ).

All tested MRSA isolates were susceptible to linezolid and vancomycin (Table 3). Between 2014 and 2018, daptomycin resistance was detected in 12 isolates. Clindamycin resistance among MRSA isolates decreased by 24.1\% between 2014 
(65.4\%, n=221/338) and 2018 (41.3\%, n=290/702). Although erythromycin and ciprofloxacin resistance has slowly decreased since 2014 , resistance remains high $\mathbf{7 5 . 6 \%}$ and $71.7 \%$ in 2018 , respectively).

Table 3: Methicillin-resistant Staphylococcus aureus bloodstream infections data, Canada, 2014-2018

\begin{tabular}{|c|c|c|c|c|c|c|c|c|c|c|}
\hline \multirow{2}{*}{$\begin{array}{c}\text { MRSA BSI } \\
\text { data }\end{array}$} & \multicolumn{10}{|c|}{ Year } \\
\hline & \multicolumn{2}{|c|}{2014} & \multicolumn{2}{|c|}{2015} & \multicolumn{2}{|c|}{2016} & \multicolumn{2}{|c|}{2017} & \multicolumn{2}{|c|}{2018} \\
\hline \multicolumn{11}{|c|}{ Number of infections and incidence rates } \\
\hline $\begin{array}{l}\text { Number of MRSA } \\
\text { bloodstream } \\
\text { infections }\end{array}$ & & 448 & & 488 & & 604 & & 606 & & 767 \\
\hline $\begin{array}{l}\text { Rate per } \\
1,000 \text { patient } \\
\text { admissions }\end{array}$ & & 0.48 & & 0.51 & & 0.61 & & 0.61 & & 0.77 \\
\hline $\begin{array}{l}\text { Rate per } 10,000 \\
\text { patient-days }\end{array}$ & & 0.66 & & 0.7 & & 0.84 & & 0.84 & & 1.05 \\
\hline $\begin{array}{l}\text { Number of } \\
\text { reporting } \\
\text { hospitals }\end{array}$ & & 62 & & 63 & & 64 & & 65 & & 62 \\
\hline \multicolumn{11}{|c|}{ All-cause mortality rate } \\
\hline $\begin{array}{l}\text { Number of } \\
\text { deaths }\end{array}$ & & 106 & & 95 & & 111 & & 99 & & 144 \\
\hline $\begin{array}{l}\text { All-cause } \\
\text { mortality rate per } \\
100 \text { cases }\end{array}$ & & 24.9 & & 20.5 & & 19.1 & & 16.4 & & 18.8 \\
\hline $\begin{array}{l}\text { Antimicrobial } \\
\text { resistance }^{b}\end{array}$ & $n$ & $\%$ & $\mathrm{n}$ & $\%$ & $n$ & $\%$ & $n$ & $\%$ & $n$ & $\%$ \\
\hline Erythromycin & 305 & 85.0 & 318 & 81.7 & 418 & 78.7 & 455 & 81.0 & 531 & 75.6 \\
\hline Ciprofloxacin & 54 & 87.1 & 73 & 81.1 & 411 & 77.4 & 432 & 76.9 & 504 & 71.7 \\
\hline Clindamycin & 221 & 65.4 & 213 & 54.8 & 230 & 43.3 & 239 & 42.5 & 290 & 41.3 \\
\hline Tetracycline & 18 & 5.0 & 14 & 3.6 & 31 & 5.8 & 35 & 6.2 & 50 & 7.1 \\
\hline $\begin{array}{l}\text { Trimethoprim/ } \\
\text { sulfamethoxazole }\end{array}$ & 6 & 1.7 & 6 & 1.5 & 11 & 2.1 & 8 & 1.4 & 14 & 2.0 \\
\hline Rifampin & 2 & 0.6 & 2 & 0.5 & 10 & 1.9 & 9 & 1.6 & 6 & 0.9 \\
\hline Tigecycline & 7 & 1.9 & 3 & 0.8 & 0 & 0.0 & 0 & 0.0 & 0 & 0.0 \\
\hline Daptomycin & 1 & 0.3 & 1 & 0.3 & 5 & 0.9 & 5 & 0.9 & 0 & 0.0 \\
\hline $\begin{array}{l}\text { Total number of } \\
\text { isolates tested }\end{array}$ & 359 & $\mathrm{~N} / \mathrm{A}$ & 389 & $\mathrm{~N} / \mathrm{A}$ & 531 & $\mathrm{~N} / \mathrm{A}$ & 562 & $\mathrm{~N} / \mathrm{A}$ & 702 & N/A \\
\hline $\begin{array}{l}\text { Abbreviations: MRSA } \\
\text { loodstream infection } \\
\text { All MRSA isolates fro } \\
\text { susceptible to linezoli } \\
\text { Based on the numbe } \\
\text { In some years, the n } \\
\text { solates tested for clin } \\
\text {-iprofloxacin }\end{array}$ & $\begin{array}{l}\text { of cas } \\
\text { mber }\end{array}$ & $\begin{array}{l}\text { illin-res } \\
\text { tot app } \\
\text { to } 201 \\
\text { ancomy } \\
\text { es with } \\
\text { of isolat } \\
\text { n, and } 6\end{array}$ & $\begin{array}{l}\text { stant } S \\
\text { icable } \\
8 \text { subm } \\
\text { cin } \\
\text { associa } \\
\text { es teste } \\
2 \text { teste }\end{array}$ & $\begin{array}{l}\text { aureus; } \\
\text { ted to } \\
\text { ed } 30-c \\
\text { for re } \\
\text { for cip }\end{array}$ & $\begin{array}{l}\text { ay ou } \\
\text { stanc }\end{array}$ & $\begin{array}{l}\text { BSI, me } \\
\text { I Microl } \\
\text { ome da } \\
\text { varied } \\
\text { in; in 2 }\end{array}$ & $\begin{array}{l}\text { thicillin } \\
\text { oiology } \\
\text { ta } \\
\text { by antik } \\
015,90\end{array}$ & $\begin{array}{l}\text { Lesistan } \\
\text { Labora } \\
\text { iotic: In } \\
\text { solates }\end{array}$ & $\begin{array}{l}\text { S. au } \\
\text { ory we } \\
2014, \\
\text { testec }\end{array}$ & \\
\hline
\end{tabular}

Since 2015, community-associated MRSA10 (USA300) has remained the predominant MRSA strain type $(46.6 \%$ in 2018 , $\mathrm{n}=327 / 702$ ) while the proportion of community-associated MRSA2 (USA100/800) continued to decrease, representing less than one-third of all strain types identified in 2018 (Table S2.2).

\section{Vancomycin-resistant Enterococci bloodstream infections}

From 2014 to 2018 , VRE BSI rates have increased by $143 \%$ from 0.14 to 0.34 infections per 10,000 patient-days $(p=0.023$ ) (Table 4). The VRE BSI rates were highest in Central and Western Canada (0.42 and 0.33 infections per 10,000 patient-days respectively) with few VRE BSIs reported in Eastern Canada (0.01 infections per 10,000 patient-days) (Table S3.1). VRE infection was predominantly a healthcare-associated infection, with $95.2 \%$ of VRE BSIs reported from 2014 to 2018 acquired in a healthcare facility (Table S3.2). All-cause mortality remained high (31.4\%) from 2014 to 2018.

\section{Table 4: Vancomycin-resistant Enterococci bloodstream infections data, Canada, 2014-2018}

\begin{tabular}{|c|c|c|c|c|c|c|c|c|c|c|}
\hline \multirow{2}{*}{ VRE BSI data } & \multicolumn{10}{|c|}{ Year } \\
\hline & \multicolumn{2}{|c|}{2014} & \multicolumn{2}{|c|}{2015} & \multicolumn{2}{|c|}{2016} & \multicolumn{2}{|c|}{2017} & \multicolumn{2}{|c|}{2018} \\
\hline \multicolumn{11}{|c|}{ Number of infections and incidence rates } \\
\hline $\begin{array}{l}\text { Number of VRE } \\
\text { bloodstream } \\
\text { infections }\end{array}$ & & 91 & & 89 & & 121 & & 155 & & 243 \\
\hline $\begin{array}{l}\text { Rate per } 1,000 \\
\text { patient admissions }\end{array}$ & & 0.10 & & 0.10 & & 0.13 & & 0.16 & & 0.24 \\
\hline $\begin{array}{l}\text { Rate per } 10,000 \\
\text { patient-days }\end{array}$ & & 0.14 & & 0.14 & & 0.18 & & 0.23 & & 0.34 \\
\hline $\begin{array}{l}\text { Number of reporting } \\
\text { hospitals }\end{array}$ & & 60 & & 57 & & 59 & & 59 & & 62 \\
\hline $\begin{array}{l}\text { Antimicrobial } \\
\text { resistance of } \\
\text { Enterococcus } \\
\text { faecium isolates }\end{array}$ & $\mathrm{n}$ & $\%$ & $\mathrm{n}$ & $\%$ & $\mathrm{n}$ & $\%$ & $\mathrm{n}$ & $\%$ & $N / n$ & $\%$ \\
\hline Ampicillin & 70 & 100.0 & 75 & 100.0 & 91 & 100.0 & 116 & 100.0 & 181 & 100.0 \\
\hline Chloramphenicol & 0 & 0.0 & 0 & 0.0 & 2 & 2.2 & 11 & 9.5 & 4 & 2.2 \\
\hline Ciprofloxacin & 70 & 100.0 & 75 & 100.0 & 91 & 100.0 & 116 & 100.0 & 181 & 100.0 \\
\hline Daptomycin ${ }^{a}$ & 0 & 0.0 & 0 & 0.0 & 7 & 7.7 & 10 & 8.6 & 12 & 6.6 \\
\hline Erythromycin & 65 & 92.9 & 72 & 96.0 & 83 & 91.2 & 108 & 93.1 & 173 & 95.6 \\
\hline $\begin{array}{l}\text { High-level } \\
\text { gentamicin }\end{array}$ & 7 & 10.0 & 6 & 8.0 & 12 & 13.2 & 45 & 38.8 & 77 & 42.5 \\
\hline Levofloxacin & 70 & 100.0 & 75 & 100.0 & 91 & 100.0 & 116 & 100.0 & 179 & 98.9 \\
\hline Linezolid & 0 & 0.0 & 0 & 0.0 & 1 & 1.1 & 0 & 0.0 & 2 & 1.1 \\
\hline Nitrofurantoin & 15 & 21.4 & 25 & 33.3 & 35 & 38.5 & 52 & 44.8 & 55 & 30.4 \\
\hline Penicillin & 70 & 100.0 & 75 & 100.0 & 91 & 100.0 & 116 & 100.0 & 181 & 100.0 \\
\hline Synercid & 5 & 7.1 & 2 & 2.7 & 9 & 9.9 & 8 & 6.9 & 18 & 9.9 \\
\hline Rifampicin & 54 & 77.1 & 71 & 94.7 & 85 & 93.4 & 110 & 94.8 & 163 & 90.1 \\
\hline $\begin{array}{l}\text { High-level } \\
\text { streptomycin }\end{array}$ & 29 & 41.4 & 27 & 36.0 & 32 & 35.2 & 39 & 33.6 & 60 & 33.1 \\
\hline Tetracycline & 38 & 54.3 & 44 & 58.7 & 46 & 50.5 & 66 & 56.9 & 108 & 59.7 \\
\hline Tigecycline & 2 & 2.9 & 0 & 0.0 & 0 & 0.0 & 0 & 0.0 & 1 & 0.6 \\
\hline Vancomycin & 70 & 100.0 & 74 & 98.7 & 88 & 96.7 & 111 & 95.7 & 176 & 97.2 \\
\hline $\begin{array}{l}\text { Total number of } \\
\text { isolates tested }\end{array}$ & 70 & N/A & 75 & N/A & 91 & N/A & 116 & N/A & 181 & N/A \\
\hline
\end{tabular}


High-level gentamycin resistance among VRE BSI isolates increased from $10.0 \%$ to $42.5 \%$ from 2014 to 2018 while daptomycin non-susceptibility was first identified in 2016 (7.7\%) and remained stable for 2017 and 2018 (Table 4). Since 2014, the majority (95.7\%-100\%) of VRE BSI isolates were identified as Enterococcus faecium. However, in 2018, three E. faecalis VRE BSI isolates were identified (Table S3.3). Among E. faecium isolates, sequence type 1478 was first identified in 2013 (data not shown) and increased from $4.0 \%$ (for 2014) to $38.7 \%$ (for 2018).

\section{Carbapenemase-producing Enterobacteriaceae}

From 2014 to 2018, the CPE infection rates remained low and stable (0.04 infections per 10,000 patient-days), while a nearly five-fold increase in colonization rates was observed $(p=0.014)$ (Table 5). Regionally, the majority of CPE infections $(51.8 \%$ $n=57 / 110$ ) were identified in Western Canada, followed by Central Canada (45.5\%, $n=50 / 110)$ and few CPE infections were identified in Eastern Canada (2.7\%, n=3/110) (Table S4.1).

Table 5: Carbapenemase-producing Enterobacteriaceae data, Canada, 2014-2018

\begin{tabular}{|c|c|c|c|c|c|c|c|c|c|c|}
\hline \multirow{2}{*}{ CPE data } & \multicolumn{10}{|c|}{ Year } \\
\hline & \multicolumn{2}{|c|}{2014} & \multicolumn{2}{|c|}{2015} & \multicolumn{2}{|c|}{2016} & \multicolumn{2}{|c|}{2017} & \multicolumn{2}{|c|}{2018} \\
\hline \multicolumn{11}{|c|}{ Number of infections and incidence rates } \\
\hline $\begin{array}{l}\text { Number of CPE } \\
\text { infections }\end{array}$ & & 22 & & 19 & & 20 & & 19 & & 30 \\
\hline $\begin{array}{l}\text { Infection rate } \\
\text { per } 1,000 \text { patient } \\
\text { admissions }\end{array}$ & & 0.03 & & 0.02 & & 0.02 & & 0.02 & & 0.03 \\
\hline $\begin{array}{l}\text { Infection rate } \\
\text { per } 10,000 \\
\text { patient-days }\end{array}$ & & 0.04 & & 0.03 & & 0.03 & & 0.03 & & 0.04 \\
\hline $\begin{array}{l}\text { Number of CPE } \\
\text { colonizations }\end{array}$ & & 23 & & 36 & & 76 & & 108 & & 130 \\
\hline $\begin{array}{l}\text { Colonization rate } \\
\text { per } 1,000 \text { patient } \\
\text { admissions }\end{array}$ & & 0.03 & & 0.04 & & 0.08 & & 0.12 & & 0.14 \\
\hline $\begin{array}{l}\text { Colonization } \\
\text { rate per } 10,000 \\
\text { patient-days }\end{array}$ & & 0.04 & & 0.05 & & 0.12 & & 0.16 & & 0.19 \\
\hline $\begin{array}{l}\text { Number of } \\
\text { reporting } \\
\text { hospitals }\end{array}$ & & 57 & & 58 & & 57 & & 58 & & 59 \\
\hline \multicolumn{11}{|c|}{ Drugs tested for antimicrobial resistance } \\
\hline Antibiotics $^{b}$ & $\mathrm{n}$ & $\%$ & $\mathrm{n}$ & $\%$ & $\mathrm{n}$ & $\%$ & $\mathrm{n}$ & $\%$ & $\mathrm{n}$ & $\%$ \\
\hline $\begin{array}{l}\text { Piperacillin- } \\
\text { Tazobactam }\end{array}$ & 59 & 89.4 & 75 & 98.7 & 117 & 95.9 & 159 & 96.4 & 209 & 95.0 \\
\hline Cefotaxime & 59 & 88.1 & 71 & 87.7 & 147 & 90.7 & 168 & 89.8 & 196 & 86.3 \\
\hline Ceftazidime & 59 & 88.1 & 69 & 85.2 & 139 & 85.8 & 160 & 85.6 & 191 & 84.1 \\
\hline Meropenem & 63 & 94.0 & 69 & 85.2 & 140 & 86.4 & 159 & 85.0 & 198 & 87.2 \\
\hline Ciprofloxacin & 49 & 73.1 & 64 & 79.0 & 134 & 82.7 & 138 & 73.8 & 157 & 69.2 \\
\hline Amikacin & 17 & 25.4 & 22 & 27.2 & 42 & 25.9 & 32 & 17.1 & 42 & 18.5 \\
\hline Gentamicin & 34 & 50.7 & 40 & 49.4 & 62 & 38.3 & 64 & 34.2 & 78 & 34.4 \\
\hline Tobramycin & 42 & 62.7 & 40 & 49.4 & 75 & 46.3 & 71 & 38.0 & 100 & 44.1 \\
\hline $\begin{array}{l}\text { Trimethoprim- } \\
\text { sulfamethoxazole }\end{array}$ & 45 & 67.2 & 59 & 72.8 & 103 & 63.6 & 113 & 60.4 & 142 & 62.6 \\
\hline
\end{tabular}

Table 5: Carbapenemase-producing Enterobacteriaceae data, Canada, 2014-2018 (continued)

\begin{tabular}{|c|c|c|c|c|c|c|c|c|c|c|}
\hline \multirow{2}{*}{ CPE data } & \multicolumn{10}{|c|}{ Year } \\
\hline & \multicolumn{2}{|c|}{2014} & \multicolumn{2}{|c|}{2015} & \multicolumn{2}{|c|}{2016} & \multicolumn{2}{|c|}{2017} & \multicolumn{2}{|c|}{2018} \\
\hline \multicolumn{11}{|c|}{ Drugs tested for antimicrobial resistance (continued) } \\
\hline Antibiotics $^{b}$ & $\mathrm{n}$ & $\%$ & $\mathrm{n}$ & $\%$ & $\mathrm{n}$ & $\%$ & $n$ & $\%$ & $\mathrm{n}$ & $\%$ \\
\hline Tigecycline & 11 & 16.4 & 13 & 16.0 & 32 & 19.8 & 18 & 9.6 & 29 & 12.8 \\
\hline $\begin{array}{l}\text { Total number of } \\
\text { isolates tested }\end{array}$ & 67 & $\mathrm{~N} / \mathrm{A}$ & 81 & N/A & 162 & $\mathrm{~N} / \mathrm{A}$ & 187 & $\mathrm{~N} / \mathrm{A}$ & 227 & $\mathrm{~N} / \mathrm{A}$ \\
\hline \multicolumn{11}{|c|}{ Carbapenemases identified } \\
\hline Carbapenemases & $n$ & $\%$ & $\mathrm{n}$ & $\%$ & $\mathrm{n}$ & $\%$ & $\mathrm{n}$ & $\%$ & $\mathrm{n}$ & $\%$ \\
\hline KPC & 33 & 49.3 & 28 & 34.6 & 84 & 51.6 & 86 & 46.0 & 120 & 52.9 \\
\hline NDM & 15 & 22.4 & 28 & 34.6 & 44 & 27.2 & 53 & 28.3 & 57 & 24.1 \\
\hline OXA-48 & 5 & 7.5 & 13 & 16.0 & 21 & 13.0 & 33 & 17.6 & 30 & 13.2 \\
\hline $\mathrm{SME}^{\mathrm{e}}$ & 5 & 7.5 & 3 & 3.7 & 4 & 2.5 & 2 & 1.1 & 4 & 1.8 \\
\hline NDM/OXA-48 & 2 & 3.0 & 1 & 1.2 & 4 & 2.5 & 5 & 2.7 & 6 & 2.6 \\
\hline GES & 1 & 1.5 & 5 & 6.2 & 0 & 0.0 & 1 & 0.5 & 1 & 0.4 \\
\hline IMP & 1 & 1.5 & 0 & 0.0 & 0 & 0.0 & 0 & 0.0 & 3 & 1.3 \\
\hline NMC & 2 & 3.0 & 0 & 0.0 & 2 & 1.2 & 4 & 2.1 & 2 & 0.9 \\
\hline VIM & 3 & 4.5 & 3 & 3.7 & 2 & 1.2 & 3 & 1.6 & 2 & 0.9 \\
\hline Other & 0 & 0.0 & 0 & 0.0 & 1 & 0.6 & 0 & 0.0 & 2 & 0.9 \\
\hline $\begin{array}{l}\text { Total number of } \\
\text { isolates tested }\end{array}$ & 67 & 100 & 81 & 100 & 162 & 100 & 187 & 100 & 227 & 100 \\
\hline
\end{tabular}

Abbreviations: CPE, carbapenemase-producing Enterobacteriaceae; GES, Guiana extended-spectrum ß-lactamase; IMP, active-on-imipenem; KPC, Klebsiella pneumoniae carbapenemase; NDM, New Delhi metallo-ß-lactamase; OXA-48, Oxacillinase-48; N/A, not applicable; NMC, not metalloenzyme carbapenemase; SME, Serratia marcescens enzymes; VIM, Verona integron-encoded metallo-ß-lactamase

Includes data for all CPE isolates submitted

${ }^{b}$ All isolates were resistant to ampicillin, and all but one to cefazolin. All

carbapenemase-producing organism isolates were screened for the mcr-type gene which is an acquired gene associated with colistin resistance

c The denominator for this drug was adjusted as MIC values were not given in all cases due to vitek algorithms

${ }^{d}$ Total number reflects the number of isolates tested for each of the antibiotics listed above e Only found in Serratia marcescens

Whereas, the majority of CPE colonizations $(80.7 \%, n=301 / 373)$ were identified in Central Canada, followed by Western Canada (19.3\%, n=72/373), while no colonizations were reported in Eastern Canada (Table S4.2). Thirty-day all-cause mortality was $14.8 \%(n=16 / 108)$ among CPE-infected patients and $26.7 \%$ $(n=8 / 30)$ among those with CPE bacteremia. Among all CPE cases reported from 2014 to 2018, 41.3\% ( $n=203 / 492)$ reported travel outside of Canada and of those, $86.1 \%(n=161 / 187)$ received medical care while abroad.

From 2014 to 2018, reductions in antimicrobial resistance for CPE isolates were observed for amikacin, gentamicin, and tobramycin while all others remained stable (Table 5). The predominant carbapenemases identified in Canada were Klebsiella pneumoniae carbapenemase (KPC), New Delhi metallo-ß-lactamase (NDM), and Oxacillinase-48 (OXA-48); however, the distribution of carbapenemases varies by region with NDM dominant in Western Canada (59.1\%, $n=101 / 171)$ and KPC dominant in Central Canada (60.4\%, n=330/546). Among submitted isolates from 2014 to 2018 , the most 
commonly identified carbapenemase-producing pathogens were K. pneumoniae (25.4\%-37.3\%), E. coli (14.7\%-29.9\%), and Enterobacter cloacae complex (11.1\%-18.9\%) (Table S5).

\section{Antibiogram}

From 2015 to 2018, E. coli antibiotic non-susceptibility rates among all specimen types tested remained relatively stable (Table 6). In 2018, the antibiotics with the highest non-susceptibility rates were ampicillin (43.0\%), trimethoprim/ sulfamethoxazole (22.6\%), ciproflaxin (19.6\%) and amoxicillin-clavulanate (16.3\%). Carbapenem resistance remained low: meropenem ( $0.4 \%$ non-susceptible) and ertapenem $(0.2 \%)$.

\section{Discussion}

In this surveillance we have shown that infection rates in Canada (including both HA and CA cases) reported via CNISP decreased for CDI (12.5\% decrease from 2015 to 2018) but increased for MRSA BSI and VRE BSI (59\% and $143 \%$, respectively, from 2014 to 2018). Although CPE infection rates remained low, colonizations increased nearly five-fold from 2014 to 2018. Globally, the overall burden of CDI has been decreasing since 2004, with Canadian rates following a similar pattern. The $\mathrm{CDI}$ rates are higher in North America compared with other regions (15).

\section{Table 6: Number of Escherichia coli isolates tested and percent non-susceptible, 2015-2018}

\begin{tabular}{|c|c|c|c|c|c|c|c|c|}
\hline \multirow{3}{*}{ Antibiotic classes } & \multicolumn{8}{|c|}{ Year } \\
\hline & \multicolumn{2}{|c|}{2015} & \multicolumn{2}{|c|}{2016} & \multicolumn{2}{|c|}{2017} & \multicolumn{2}{|c|}{2018} \\
\hline & $\begin{array}{l}\text { \# isolates } \\
\text { tested }\end{array}$ & $\begin{array}{c}\% \text { non- } \\
\text { susceptible }\end{array}$ & $\begin{array}{l}\text { \# isolates } \\
\text { tested }\end{array}$ & $\begin{array}{c}\% \text { non- } \\
\text { susceptible }\end{array}$ & $\begin{array}{l}\text { \# isolates } \\
\text { tested }\end{array}$ & $\begin{array}{c}\% \text { non- } \\
\text { susceptible }\end{array}$ & $\begin{array}{l}\text { \# isolates } \\
\text { tested }\end{array}$ & $\begin{array}{c}\% \text { non- } \\
\text { susceptible }\end{array}$ \\
\hline \multicolumn{9}{|c|}{ Penicillins and penicillin combinations } \\
\hline Ampicillin & 66,756 & 43.7 & 52,198 & 44.0 & 66,583 & 40.2 & 62,983 & 39.6 \\
\hline Amoxicillin clavulanate & 56,200 & 16.8 & 43,516 & 16.6 & 60,428 & 14.9 & 58,243 & 16.7 \\
\hline Piperacillin-Tazobactam & 59,085 & 5.3 & 49,956 & 4.7 & 61,723 & 4.5 & 59,770 & 5.2 \\
\hline \multicolumn{9}{|l|}{ Cephalosporins } \\
\hline Cephalothin & ND & N/A & 17,504 & 46.9 & 9,072 & 42.2 & 1,877 & 12.1 \\
\hline $\begin{array}{l}\text { Cefazolin (for systemic } \\
\text { use) }\end{array}$ & 40,291 & 19.1 & 23,048 & 25.2 & 29,347 & 19.3 & 40,440 & 24.9 \\
\hline $\begin{array}{l}\text { Cefazolin (marker for oral } \\
\text { use) }\end{array}$ & ND & N/A & 19,300 & 22.7 & 9,078 & 28.6 & 11,902 & 15.2 \\
\hline Cefuroxime & ND & N/A & 496 & 7.0 & 2,363 & 16.2 & 5,783 & 31.1 \\
\hline Cefoxitin & ND & N/A & 26,162 & 9.4 & 14,174 & 6.5 & 22,076 & 7.1 \\
\hline Ceftriaxone & 57,215 & 8.5 & 42,157 & 9.2 & 56,138 & 7.9 & 61,377 & 9.4 \\
\hline Cefotaxime (pediatric) & ND & N/A & 3,870 & 8.6 & 578 & 3.0 & 389 & 10.3 \\
\hline \multicolumn{9}{|l|}{ Carbapenems } \\
\hline Ertapenem & ND & N/A & 34,501 & 0.5 & 38,789 & 0.4 & 36,129 & 0.3 \\
\hline Imipenem & ND & N/A & 31,535 & 0.3 & 28,037 & 0.4 & 11,971 & 0.8 \\
\hline Meropenem & 44,299 & 0.5 & 37,875 & 0.1 & 41,955 & 0.1 & 58,491 & 0.3 \\
\hline \multicolumn{9}{|l|}{ Fluoroquinolones } \\
\hline Ciprofloxacin & 64,548 & 18.4 & 52,179 & 18.9 & 66,396 & 18.3 & 62,267 & 19.8 \\
\hline Levofloxacin & ND & N/A & 10,550 & 19.4 & ND & N/A & ND & $\mathrm{N} / \mathrm{A}$ \\
\hline \multicolumn{9}{|l|}{ Aminoglycosides } \\
\hline Gentamicin & 51,714 & 7.7 & 52,207 & 8.0 & 64,351 & 7.5 & 62,992 & 8.5 \\
\hline Tobramycin & 40,654 & 7.4 & 47,441 & 8.9 & 61,572 & 8.1 & 61,640 & 7.4 \\
\hline Amikacin & ND & N/A & 34,905 & 0.1 & 35,095 & 0.2 & 23,672 & 0.6 \\
\hline \multicolumn{9}{|l|}{ Other } \\
\hline $\begin{array}{l}\text { Trimethoprim/ } \\
\text { sulfamethoxazole }\end{array}$ & 66,760 & 22.3 & 48,672 & 23.1 & 66,442 & 20.8 & 44,001 & 22.7 \\
\hline Nitrofurantoin & 62,020 & 4.9 & 39,943 & 2.9 & 45,356 & 2.8 & 47,985 & 3.0 \\
\hline Fosfomycin & ND & N/A & 12,911 & 0.1 & 17,584 & 2.5 & 15,776 & 0.8 \\
\hline Number of hospitals ${ }^{c}$ & & 21 & & 50 & & 70 & & 65 \\
\hline
\end{tabular}

Abbreviations: ND data not collected; N/A, not applicable

all patient types include inpatients and outpatients, all specimen types include urine, blood and any other source (e.g. wound, respiratory, etc.)

b Antibiogram data collection was a pilot project in 2015

' Includes hospitals that do and do not participate in Canadian Nosocomial Infection Surveillance Program 
Decreasing moxifloxacin resistance (11.1\% in 2018) in Canada was associated with declining ribotype 027 prevalence, and remained lower than previously published data in Europe (35.8\%) and the US (38.0\%) (16-18). Estimates of HA-CDI rates from tertiary care hospitals in Europe and Australia showed lower rates of HA-CDI compared with Canada $(19,20)$. Decreased Canadian $C D I$ rates suggest improvements in infection prevention and control practices in hospitals, such as hand hygiene compliance, environmental cleaning, antibiotic stewardship and increased awareness of infection (21).

An increase in the rates of MRSA BSI, attributed to the increase in CA-MRSA BSI rates, is raising concerns as these infections are associated with a mortality rate higher than $20 \%$ among admitted patients (22). As MRSA resistance trends are closely tied to the prevalence of epidemic strains, the decrease in the proportion of strain types that are identified as CMRSA2 is driving down clindamycin resistance among isolates (23). The incidence of MRSA BSI in 2017 was lower than the rates reported by South Korea (0.84 versus 1.6 infections per 10,000 patient days) (24). In a US study, reported medium-sized US hospital-onset MRSA BSI rates between 2016 and 2017 were slightly lower than were healthcare-associated 2017 MRSA BSI rates in Canada (0.45 versus 0.47 infections per 10,000 patientdays), but rates for large US hospitals were higher ( 0.54 versus 0.42 infections per 10,000 patient-days) (25).

The increase in VRE BSI rates in Canada is a concerning trend as hospitalized patients with VRE bacteremia have a higher risk of mortality and longer length of stay when compared with vancomycin-susceptible Enterococcus bacteremia (26). This increase may be due to differences in infection control practices across acute care hospitals, with some hospitals discontinuing the practice of admission screening and use of contact precautions for infected and colonized patients (27).

Laboratory surveillance of VRE isolates revealed an emerging strain, ST1478, associated with daptomycin non-susceptibility and high-level gentamicin resistance. First identified in Australia $(28,29)$, pstS negative sequence types emerged in Canada primarily through the identification of ST1478 and may be associated with increased rates of VRE BSI (30). Further investigation is ongoing to understand the emergence and transmission dynamics of this novel strain in Canada.

Defined as antibiotics of last resort by the World Health Organization, carbapenems are now threatened by the emergence of carbapenem-resistant organisms (31). While observed CPE rates are low in Canada, colonizations increased nearly five-fold from 2014 to 2018 . Changes in screening practices may have contributed to the increase in reported colonization rates and will be collected moving forward (13). National surveillance suggests increases in CPE are driven by local nosocomial transmission as well as travel and healthcare from endemic areas, as has been reported in Ontario (32). There is continued need for the coordination of infection control measures and surveillance to prevent further transmission of CPE in Canadian acute care hospitals.

Antibiogram data has confirmed that antibiotic susceptibility to E. coli has changed minimally in Canada from 2014-2018. Standardized, routine reporting on AMR data through CNISP contributes to crucial international collaborative initiatives such as the World Health Organization Global Antimicrobial Resistant Surveillance System (33).

Consistent and uniform surveillance that helps to inform infection control practices and antimicrobial stewardship programs are essential to reducing the rates of infection and AMR, both of which cause substantial increases in healthcare costs, morbidity and mortality (15).

\section{Strengths and limitations}

The main strength of CNISP surveillance data is the active collection of standardized, detailed, epidemiologic and laboratory-linked data from 70 sentinel hospitals across Canada. However, it is primarily large, tertiary acute care hospitals that participate in CNISP, and these hospitals may not fully represent the general Canadian inpatient population. The CNISP is currently undergoing a recruitment process in order to increase representativeness and coverage of Canadian inpatient beds, especially in Northern, rural community and indigenous populations.

The CNISP data, although standardized, may be sensitive to changes in hospital participation infection prevention and control practices and the application of surveillance definitions.

\section{Next steps}

Continued recruitment of hospitals into the CNISP network with a 2020 goal of $33 \%$ national acute-care bed coverage from all ten provinces and three territories will improve the quality and representativeness of $\mathrm{HAl}$ estimates in Canada. To address gaps in surveillance data, detailed hospital screening practice surveys will be conducted annually to better interpret changes in $\mathrm{HAl}$ rates. Additionally, steps have been taken to gauge interest in the surveillance of non-acute care settings within the CNISP network such as long-term care facilities. Epidemiologic and laboratory-led working groups were also formed to investigate new and emerging pathogens such as Candida auris and VRE BSI ST1478. Lastly, future CNISP antibiogram data aims to report on a broader range of patient and specimen types as well as reporting resistance data on $K$. pneumoniae, pseudomonas, acinetobacter and $S$. aureus.

\section{Conclusion}

Ongoing efforts to prevent HAls, including AROs, and to reduce AMR in Canadian acute-care hospitals require standardized surveillance and consistent infection prevention and control practices. Data presented in this article indicate rates of 
MRSA BSI, VRE BSI and CPE colonizations increased substantially between 2014 and 2018 while rates of CDI decreased. These findings indicate a need for continued vigilance to prevent morbidity and mortality attributable to HAls and AROs in the inpatient population. As new pathogens emerge, and resistance to last-resort antibiotics is identified, PHAC's continued partnership with acute-care hospitals and collaboration with provincial, territorial and international partners in infection prevention and control as well as antimicrobial stewardship are essential to reducing the burden of HAls and AROs in Canada.

\section{Authors' statement}

Canadian Nosocomial Infection Surveillance Program hospitals provided expertise in the development of protocols in addition to epidemiological data and lab isolates. National Microbiology Laboratory completed the laboratory analyses and contributed to the interpretation and revision of the paper. Epidemiologists from Public Health Agency of Canada were responsible for the conception, analysis, interpretation, drafting, and revision of the paper.

\section{Conflict of interest}

None.

\section{Acknowledgements}

We gratefully acknowledge the contribution of the physicians, epidemiologists, infection control practitioners and laboratory staff at each participating hospital: Vancouver General Hospital (VGH), Vancouver, British Columbia (BC); Richmond General Hospital, Richmond, BC; UBC Hospital, Vancouver, BC; Lion's Gate, North Vancouver, BC; Powell River General Hospital, Powell River, BC; Sechelt Hospital (formerly St. Mary's), Sechelt, BC; Squamish General Hospital, Squamish, BC; Peter Lougheed Centre, Calgary, Alberta (AB); Rockyview General Hospital, Calgary, $A B$; South Health Campus, Calgary, AB; Foothills Medical Centre, Calgary, AB; Alberta Children's Hospital, Calgary, $A B$; University of Alberta Hospital, Edmonton, $A B$; Stollery Children's Hospital, Edmonton, $A B$; Health Sciences Centre-Winnipeg, Winnipeg, Manitoba (MB); University of Manitoba Children's Hospital, Winnipeg, MB; Children's Hospital of Western Ontario, London, Ontario (ON); Victoria Hospital, London, ON; University Hospital, London, ON; Toronto General Hospital, Toronto, ON; Toronto Western Hospital, Toronto, ON; Princess Margaret, Toronto, ON; Mount
Sinai Hospital, Toronto, ON; Bridgepoint Active Healthcare, Toronto, ON; Sunnybrook Hospital, Toronto, ON; Kingston General Hospital, Kingston, ON; SMBD - Jewish General Hospital, Montréal, Quebec (QC); The Moncton Hospital, Moncton, New Brunswick (NB); Halifax Infirmary, Halifax, Nova Scotia (NS); Victoria General, Halifax, NS; Rehabilitation Centre, Halifax, NS; Veterans Memorial Building, Halifax, NS; Dartmouth General Hospital, Halifax, NS; IWK Health Centre, Halifax, NS; Hospital for Sick Children, Toronto, ON; Montreal Children's Hospital, Montréal, QC; Royal University Hospital, Saskatoon, Saskatchewan (SK); St. Paul's Hospital, Saskatoon, SK; General Hospital \& Miller Centre, St. John's, Newfoundland and Labrador (NL); Burin Peninsula Health Care Centre, Burin, NL; Carbonear General Hospital, Carbonear, NL; Dr. G.B.

Cross Memorial Hospital, Clarenville, NL; Janeway Children's Hospital and Rehabilitation Centre, St. John's, NL; St. Clare's Mercy Hospital, St. John's, NL; McMaster Children's Hospital, Hamilton, ON; St Joseph's Healthcare, Hamilton, ON; Jurvinski Hospital and Cancer Center, Hamilton, ON; General Site, Hamilton, ON; Civic Campus, Ottawa, ON; General Campus, Ottawa, ON; University of Ottawa Heart Institute, Ottawa, ON; Hôpital Maisonneuve-Rosemont, Montréal, QC; Victoria General Hospital, Victoria, BC; Royal Jubilee, Victoria, BC; Nanaimo Regional General Hospital, Nanaimo, BC; Children's Hospital of Eastern Ontario (CHEO), Ottawa, ON; BC Women's Hospital, Vancouver, BC; Hôtel-Dieu de Québec, Québec, QC; Montreal General Hospital, Montréal, QC; Royal Victoria Hospital, Montréal, QC; Montreal Neurological Institute, Montréal, QC; North York General Hospital, Toronto, ON; Kelowna General Hospital, Kelowna, BC; Queen Elizabeth Hospital, Charlottetown, Prince Edward Island (PE); Prince County Hospital, Summerside, PE; Western Memorial Regional Hospital, Corner Brook, NL; Regina General Hospital, Regina, SK; Pasqua Hospital, Regina, SK; Sudbury Regional Hospital, Sudbury, ON; University Hospital of Northern BC, Prince George, BC.

Thank you to the staff at Public Health Agency of Canada in the Centre for Communicable Diseases and Infection Control, Ottawa, ON (J Brooks, L Pelude, R Mitchell, W Rudnick, KB Choi, A Silva, V Steele, J Cayen, C McClellan, M Hunt and L Sauvé) and the National Microbiology Laboratory, Winnipeg, MB (G Golding, M Mulvey, J Campbell, T Du, M McCracken, L Mataseje, A Bharat and D Boyd).

\section{Funding}

This work was supported by Public Health Agency of Canada. 


\section{References}

1. World Health Organization. Report on the Burden of Endemic Health Care-Associated Infection Worldwide: Clean Care is Safer Care. Geneva (CH): WHO; 2011. https://apps. who.int/iris/bitstream/handle/10665/80135/9789241501507 eng.pdf;jsessionid=B25CB6526B6547588285C99D3CD12D0 7 ? sequence $=1$

2. Public Health Agency of Canada. Canadian Nosocomial Infection Surveillance Program (CNISP): Summary Report of Healthcare Associated Infection (HAl), Antimicrobial Resistance (AMR) and Antimicrobial Use (AMU) Surveillance Data from January 1, 2013 to December 31, 2017. Ottawa (ON); PHAC: 2018. https://www.canada.ca/en/public-health/ services/publications/science-research-data/summary-repor t-healthcare-associated-infection-antimicrobial-resistance-an timicrobial-use-surveillance-data-2013-2017.html

3. Pittet D, Boyce JM, Allegranzi B, editors. Hand Hygiene: A Handbook for Medical Professionals. John Wiley \& Sons; 2017. https://books.google.ca/books?hl=en\&lr=\&id=21 rMDgAAQBAJ\&oi=fnd\&pg=PA1\&dq=healthcare + associ ated+infection\&ots $=c T A c p \mid M v 4 e \& s i g=W 3 l j N y U 1 R h G f m$ wdlq1G97zPSOZO\#v=onepage\&q=healthcare associated infection\&f=false

4. Valiquette L, Chakra CN, Laupland KB. Financial impact of health care-associated infections: when money talks. Can J Infect Dis Med Microbiol 2014;25(2):71-4. DOI PubMed

5. Mitchell R, Taylor G, Rudnick W, Alexandre S, Bush K, Forrester L, Frenette C, Granfield B, Gravel-Tropper D, Happe J, John M, Lavallee C, McGeer A, Mertz D, Pelude L, Science M, Simor A, Smith S, Suh KN, Vayalumkal J, Wong A, Amaratunga K; Canadian Nosocomial Infection Surveillance Program. Trends in health care-associated infections in acute care hospitals in Canada: an analysis of repeated point-prevalence surveys. CMAJ September 9, 2019;191(36):E981-8. DOI PubMed

6. Suetens $C$, Latour K, Kärki T, Ricchizzi E, Kinross $P$, Moro ML, Jans B, Hopkins S, Hansen S, Lyytikäinen $O$, Reilly J, Deptula A, Zingg W, Plachouras D, Monnet DL; The Healthcare-Associated Infections Prevalence Study Group. Prevalence of healthcare-associated infections, estimated incidence and composite antimicrobial resistance index in acute care hospitals and long-term care facilities: results from two European point prevalence surveys, 2016 to 2017. Euro Surveill. 2018;23(46). DOI

7. Cassini A, Plachouras D, Eckmanns T, Abu Sin M, Blank HP, Ducomble T, Haller $S$, Harder T, Klingeberg A, Sixtensson M, Velasco E, Weiß B, Kramarz P, Monnet DL, Kretzschmar ME, Suetens $C$. Burden of six healthcare-associated infections on European population health: estimating incidence-based disability-adjusted life years through a population prevalence-based modelling study. PLoS Med 2016;13(10):e1002150. DOI PubMed

8. World health Organization. Global Antimicrobial Resistance Surveillance System (GLASS) Report. Early implementation 2016-2017. Geneva (CH); WHO: 2017. https://www.who.int/ glass/resources/publications/early-implementation-report/ en/
9. The Review on Antimicrobial Resistance. Antimicrobial Resistance: Tackling a crisis for the health and wealth of nations. O'Neill J, Chair. UK: Department of Health, HM Treasury, Foreign and Commonwealth Office: 2014. https:// amr-review.org/sites/default/files/AMR\%20Review\%20 Paper\%20-\%20Tackling\%20a\%20crisis\%20for\%20the\%20 health\%20and\%20wealth\%20of\%20nations_1.pdf

10. World Health Organization. Guidelines on core components of infection prevention and control programmes at the national and acute health care facility level. Geneva $(\mathrm{CH})$; WHO: 2016. https://www.who.int/gpsc/ipc-components/en/

11. Public Health Agency of Canada. Pan-Canadian framework for action on antimicrobial resistance and antimicrobial use. Can Commun Dis Rep 2017;43(11):217-9. DOI PubMed

12. Forrester $L$, Collet JC, Mitchell R, Pelude $L$, Henderson $E$, Vayalumkal J, Leduc S, Ghahreman S, Weir C, Gravel D; CNISP Data Quality Working Group, and CNISP participating sites. How reliable are national surveillance data? Findings from an audit of Canadian methicillin-resistant Staphylococcus aureus surveillance data. Am J Infect Control 2012;40(2):102-7. DOI PubMed

13. Leduc S, Bush K, Campbell J, Cassidy K, Collet JC, Forrester L, Henderson E, Leal J, Leamon A, Pelude L, Mitchell R, Mukhi SN, Quach-Thanh C, Shurgold JH, Simmonds K; Canadian Nosocomial Infection Surveillance Program. What can an audit of national surveillance data tell us? Findings from an audit of Canadian vancomycin-resistant enterococci surveillance data. Can J Infect Control 2015;30(2):75-81. https://ipac-canada.org/photos/custom/ OldSite/cjic/vol30no2.pdf

14. Clinical Laboratory Standards Institute. M39 Analysis and Presentation of Cumulative Antimicrobial Susceptibility Test Data. 4th Edition. 2014. https://clsi.org/standards/products/ microbiology/documents/m39/

15. Ho J, Wong SH, Doddangoudar VC, Boost MV, Tse G, Ip M. Regional differences in temporal incidence of Clostridium difficile infection: a systematic review and meta-analysis. Am J Infect Control 2020;48(1):89-94. DOI PubMed

16. Freeman J, Vernon J, Pilling S, Morris K, Nicholson S, Shearman S, Longshaw C, Wilcox MH; Pan-European Longitudinal Surveillance of Antibiotic Resistance among Prevalent Clostridium difficile Ribotypes Study Group. The ClosER study: results from a three-year pan-European longitudinal surveillance of antibiotic resistance among prevalent Clostridium difficile ribotypes, 2011-2014. Clin Microbiol Infect 2018;24(7):724-31. DOI PubMed

17. Peng Z, Jin D, Kim HB, Stratton CW, Wu B, Tang YW, Sun X. Update on antimicrobial resistance in Clostridium difficile: resistance mechanisms and antimicrobial susceptibility testing. J Clin Microbiol 2017;55(7):1998-2008. DOI PubMed

18. Tenover FC, Tickler IA, Persing DH. Antimicrobial-resistant strains of Clostridium difficile from North America. Antimicrob Agents Chemother 2012;56(6):2929-32. DOl PubMed 
19. European Centre for Disease Provention and Control. Healthcare-associated infections: Clostridium difficile infections - Annual Epidemiological Report for 2016. Stockholm: ECDPC; 2018. https://www.ecdc.europa.eu/en/ publications-data/healthcare-associated-infections-clostri dium-difficile-infections-annual

20. Worth LJ, Spelman T, Bull AL, Brett JA, Richards MJ. Epidemiology of Clostridium difficile infections in Australia: enhanced surveillance to evaluate time trends and severity of illness in Victoria, 2010-2014. J Hosp Infect 2016;93(3):2805. DOI PubMed

21. Xia Y, Tunis MC, Frenette $C$, Katz K, Amaratunga K, Rose SR, House A, Quach C. Epidemiology of Clostridioides difficile infection in Canada: A six-year review to support vaccine decision-making. Can Commun Dis Rep 2019;45(7/8):191211. DOI PubMed

22. Simor $A E$, Pelude $L$, Golding G, Fernandes R, Bryce $E$, Frenette C, Gravel D, Katz K, McGeer A, Mulvey MR, Smith S, Weiss K; Canadian Nosocomial Infection Surveillance Program. Determinants of outcome in hospitalized patients with methicillin-resistant staphylococcus aureus bloodstream infection: Results from National Surveillance in Canada, 2008-2012. Infect Control Hosp Epidemiol 2016;37(4):390-7. DOI PubMed

23. Nichol KA, Adam HJ, Roscoe DL, Golding GR, Lagacé-Wiens PR, Hoban DJ, Zhanel GG; Canadian Antimicrobial Resistance Alliance. Changing epidemiology of methicillin-resistant Staphylococcus aureus in Canada. J Antimicrob Chemother 2013;68 Suppl 1:i47-55.

DOI PubMed

24. Lee H, Yoon EJ, Kim D, Jeong SH, Won EJ, Shin JH, Kim SH, Shin JH, Shin KS, Kim YA, Uh Y, Yang JW, Kim IH, Park C, Lee KJ. Antimicrobial resistance of major clinical pathogens in South Korea, May 2016 to April 2017: first one-year report from Kor-GLASS. Euro Surveill 2018;23(42)1-11. DOI PubMed

25. Fakih MG, Battjes R, Sturm L, Jones L, Groves C, Bufalino A, Hendrich A. Hospital-Onset Staphylococcus aureus Bacteremia Is A Better Measure Than MRSA Bacteremia for Assessing Infection Prevention: evaluation of 50 US Hospitals. Infect Control Hosp Epidemiol 2018;39(4):476-8. DOI PubMed

26. Prematunge C, MacDougall C, Johnstone J, Adomako K, Lam F, Robertson J, Garber G. VRE and VSE bacteremia outcomes in the era of effective VRE therapy: A systematic review and meta-analysis. Infect Control Hosp Epidemiol 2016;37(1):26-35. DOI PubMed
27. Johnstone J, Garber G, Muller M. Health care-associated infections in Canadian hospitals: still a major problem. Can Med Assoc J September 9, 2019;191(36):E977-8. DOI

28. Carter GP, Buultjens AH, Ballard SA, Baines SL, Tomita T, Strachan J, Johnson PD, Ferguson JK, Seemann T, Stinear TP, Howden BP. Emergence of endemic MLST non-typeable vancomycin-resistant Enterococcus faecium. J Antimicrob Chemother 2016;71(12):3367-71. DOI PubMed

29. van Hal SJ, Beukers AG, Timms VJ, Ellem JA, Taylor $P$, Maley MW, Newton PJ, Ferguson JK, Lee A, Chen SC, Sintchenko V. Relentless spread and adaptation of non-typeable vanA vancomycin-resistant Enterococcus faecium: a genome-wide investigation. J Antimicrob Chemother 2018;73(6):1487-91. DOI PubMed

30. Smith S, Mitchell R, Amaratunga K, Conly J, Ellison J, Embil J, Hota S, Johnstone J, McCracken M, Al-Rawahi G, Tomlinson J, Wong J, Golding G. Emergence of A Novel ST1478 VRE in Canadian Hospitals Associated with Daptomycin Non-Susceptibility and High Level Gentamicin resistance. In: AMMI Canada-CACMID Annual Conference; 2019 Apr 3-6; Ottawa, Canada. Canadian Nosocomial Infection Surveillance Program; 2019. https:// app.oxfordabstracts.com/events/662/program-app/ submission/91012

31. Public Health Agency of Canada. Canadian Antimicrobial Resistance Surveillance System - Update 2018: Executive Summary. Ottawa (ON): PHAC; modified on April 20, 2019. https://www.canada.ca/en/public-health/services/ publications/drugs-health-products/canadian-antimicrobia I-resistance-surveillance-system-2018-report-executivesummary.html

32. Kohler PP, Melano RG, Patel SN, Shafinaz S, Faheem A, Coleman BL, Green K, Armstrong I, Almohri H, Borgia S, Borgundvaag E, Johnstone J, Katz K, Lam F, Muller MP, Powis J, Poutanen SM, Richardson D, Rebbapragada A, Sarabia A, Simor A, McGeer A; Toronto Invasive Bacterial Diseases Network (TIBDN). Emergence of carbapenemase-producing Enterobacteriaceae, south-central Ontario, Canada. Emerg Infect Dis 2018;24(9):1674-82. DOl PubMed

33. World Health Organization. Global Antimicrobial Resistance Surveillance System (GLASS). Geneva (CH): WHO; 2018. https://www.who.int/glass/en/ 


\section{Appendices}

\section{Appendix A: Surveillance case definitions and eligibility criteria, 2018}

\section{Clostridioides difficile infection (CDI)}

A "primary" episode of CDI is defined as either the first episode of $C D I$ ever experienced by the patient or a new episode of CDI, which occurs greater than eight weeks after the diagnosis of a previous episode in the same patient.

\section{A patient is identified as having CDI if:}

- The patient has diarrhea or fever, abdominal pain and/or ileus AND a laboratory confirmation of a positive toxin assay or positive polymerase chain reaction (PCR) for $C$.difficile (without reasonable evidence of another cause of diarrhea) OR

- The patient has a diagnosis of pseudomembranes on sigmoidoscopy or colonoscopy (or after colectomy) or histological/pathological diagnosis of CDI OR

- The patient is diagnosed with toxic megacolon (in adult patients only)

\section{Diarrhea is defined as one of the following:}

- More watery/unformed stools in a 36-hour period

- or more watery/ unformed stools in a 24-hour period and this is new or unusual for the patient (in adult patients only)

\section{Exclusion:}

- Any patients younger than one year

- Any pediatric patients (aged one year to younger than 18 years) with alternate cause of diarrhea found (i.e. rotavirus, norovirus, enema or medication, etc.) are excluded even if $C$. difficile diagnostic test result is positive

\section{CDI case classification}

Once a patient has been identified with CDI, the infection will be classified further based on the following criteria and the best clinical judgment of the healthcare and/or infection prevention and control practitioner.

\section{Healthcare-associated (acquired in your facility) CDI case definition}

- Related to the current hospitalization

- The patient's CDI symptoms occur in your healthcare facility three or more days (or $\geq 72$ hours) after admission

- Related to a previous hospitalization

- Inpatient: The patient's CDI symptoms occur less than three days after the current admission (or less than 72 hours) AND the patient had been previously hospitalized at your healthcare facility and discharged within the previous four weeks
- Outpatient: The patient presents with CDI symptoms at your emergency room (ER) or outpatient location AND the patient had been previously hospitalized at your healthcare facility and discharged within the previous four weeks

- Related to a previous healthcare exposure at your facility

- Inpatient: The patient's CDI symptoms occur less than three days after the current admission (or less than 72 hours) AND the patient had a previous healthcare exposure at your facility within the previous four weeks

- Outpatient: The patient presents with CDI symptoms at your ER or outpatient location AND the patient had a previous healthcare exposure at your facility within the previous four weeks

Healthcare-associated (acquired in any other healthcare facility) CDI case definition

- Related to a previous hospitalization at any other healthcare facility

- Inpatient: The patient's CDI symptoms occur less than three days after the current admission (or less than 72 hours) AND the patient is known to have been previously hospitalized at any other healthcare facility and discharged/transferred within the previous four weeks

- Outpatient: The patient presents with CDI symptoms at your ER or outpatient location AND the patient is known to have been previously hospitalized at any other healthcare facility and discharged/transferred within the previous four weeks

- Related to a previous healthcare exposure at any other healthcare facility

- Inpatient: The patient's CDI symptoms occur less than three days after the current admission (or $<72$ hours) AND the patient is known to have a previous healthcare exposure at any other healthcare facility within the previous four weeks

- Outpatient: The patient presents with CDI symptoms at your ER or outpatient location AND the patient is known to have a previous healthcare exposure at any other healthcare facility within the previous four weeks

Healthcare-associated CDI but unable to determine which facility

The patient with CDI DOES meet both definitions of healthcare-associated (acquired in your facility) and healthcare-associated (acquired in any other healthcare facility), but unable to determine to which facility the case is primarily attributable to. 


\section{Community-associated CDI case definition}

- Inpatient: The patient's CDI symptoms occur less than three days (or less than 72 hours) after admission, with no history of hospitalization or any other healthcare exposure within the previous 12 weeks

- $\quad$ Outpatient: The patient presents with CDI symptoms at your ER or outpatient location with no history of hospitalization or any other healthcare exposure within the previous 12 weeks

\section{Indeterminate $C D I$ case definition}

The patient with CDI does NOT meet any of the definitions listed above for healthcare-associated or community-associated CDI. The symptom onset was more than four weeks but less than 12 weeks after the patient was discharged from any healthcare facility or after the patient had any other healthcare exposure.

\section{Methicillin-resistant Staphylococcus aureus (MRSA)}

\section{MRSA surveillance inclusion criteria}

MRSA case definition:

- Isolation of S. aureus from any body site AND

- Resistance of isolate to oxacillin AND

- Patient must be admitted to the hospital AND

- Is a "newly identified MRSA case" at a Canadian Nosocomial Infection Surveillance Program (CNISP) hospital at the time of hospital admission or identified during hospitalization.

This includes:

- $\quad$ MRSA infections identified for the first time during this hospital admission

- Infections that have been previously identified at other NON-CNISP hospitals (since we want newly identified MRSA cases at CNISP hospitals)

- Infections that have already been identified at your site but are new infections. This can only be identified if the previously identified case has another strain. This means the person was exposed again to MRSA and acquired another strain of it from another source (a new patient identifier is assigned only if confirmed with a different strain type)

- MRSA infection identified at a new (different) site in a patient with a MRSA infection identified in a previous surveillance (calendar) year AND

- Meets the criteria for MRSA infection as determined using the January 2017 Centers for Disease Control and Prevention/National Healthcare Safety Network (CDC/ NHSN) surveillance definitions for specific infections, and in accordance with the best judgment
MRSA surveillance exclusion criteria:

- MRSA infections previously identified at other CNISP sites

- Emergency, clinic, or other outpatient cases who are NOT admitted to the hospital

- Infections readmitted with MRSA (unless it is a different strain or a new/different site of MRSA infection)

Healthcare-associated (HA) case definition:

Healthcare-associated is defined as an inpatient who meets the following criteria and in accordance with the best clinical judgement of the healthcare and/or IPC practitioner:

- Exposure to any healthcare setting (including long-term care facilities or clinics) in the previous 12 months OR

- Patient is on calendar day 3 of their hospitalization

\section{Community-associated case definition:}

- MRSA identified on admission to hospital (Calendar day 1 = day of hospital admission) and/or the day after admission (day 2)

AND

- Has no previous history of the organism AND

- Has no prior hospital, long-term care admission or other exposure to a healthcare setting (rehab, clinics) in the past 12 months AND

- Has no reported use of medical devices

\section{MRSA clinical infection}

MRSA infection is determined using the 2016 CDC/NHSN surveillance definitions for specific infections, and in accordance with the best judgment of the healthcare and/or IPC practitioner. https://www.cdc.gov/nhsn/pdfs/pscmanual/17pscnosinfdef_ current.pdf

The MRSA infection would be considered HA if all elements of a CDC/NHSN site-specific infection criterion were present on or after the third calendar day of admission to the facility (the day of hospital admission is calendar day 1). The MRSA infection would be considered CA if all elements of a CDC/NHSN site-specific infection criterion were present during the two calendar days before the day of admission, the first day of admission (day 1) and/or the day after admission (day 2) and are documented in the medical record.

\section{MRSA bloodstream infection (bacteremia)}

To be considered a MRSA bloodstream infection the patient must have MRSA cultured (lab-confirmed) from at least one blood culture. 


\section{Vancomycin-resistant Enterococci (VRE)}

VRE infection case definition:

- Isolation of Enterococcus faecalis or faecium AND

- Vancomycin MIC $>8 \mu \mathrm{g} / \mathrm{ml}$ AND

- Patient is admitted to the hospital AND

- Is a "newly" identified VRE-infection at a CNISP facility at the time of hospital admission or identified during hospitalization

VRE infection is determined using the January 2017 CDC/NHSN definitions/criteria for infections, and in accordance with the best judgment of the infection prevention and control practitioner. These criteria should be met at the time of the culture that yielded VRE, or within 72 hours of the culture.

https://www.cdc.gov/nhsn/pdfs/pscmanual/17pscnosinfdef_ current.pdf

\section{Exclusion criteria:}

- Previously identified at other CNISP sites (to avoid duplicate reporting to CNISP)

- Identified through emergency, clinic, or other outpatient areas

- Readmitted with VRE (UNLESS it is a different strain)

Healthcare-associated is defined as an inpatient who meets the following criteria and in accordance with the best clinical judgement of the healthcare and/or infection prevention and control practitioner:

- Exposure to any healthcare setting (including long-term care facilities or clinics) in the previous 12 months OR

- Patient is on calendar day 3 of their hospitalization

\section{Carbapenemase-producing Enterobacteriaceae (CPE)}

Any patient admitted to a participating CNISP hospital with a hospital laboratory confirmation (and subsequent confirmation by the National Microbiology Laboratory) that tested/screened positive for a least one potential carbapenem-reduced susceptible Enterobacteriaceae, from any body site that meets the Clinical \& Laboratory Standards Institute criteria.

Carbapenems are a class of beta-lactam antibiotics with broad-spectrum activity recommended as first-line therapy for severe infections caused by certain gram negative organisms and as directed therapy for organisms that are resistant to narrower spectrum antibiotics.

Carbapenem resistance can be due to changes in the permeability of the organism to the antibiotic and/or the upregulation of efflux systems that "pump" the antibiotic out of the cell, usually concomitant with the presence of an acquired extended-spectrum beta-lactamase or AmpC enzyme or the hyperproduction of intrinsic chromosomally-located betalactamase(s). More recently, resistance is increasingly due to the acquisition of enzymes that break down the carbapenems: carbapenemases (e.g. New Delhi metallo-ß-lactamase-1, Oxacillinase-48, Klebsiella pneumoniae carbapenemase, Verona integrin-encoded metallo-ß-lactamase, active-on-imipenem, etc.). These latter subsets of carbapenem-resistant organisms are called carbapenemase-producing organisms (CPOs) and are of particular concern because of their ability to transfer resistance easily across different genera and species of bacteria. They are quickly becoming a public health problem not only because of the ability to cause healthcare acquired infections which have limited treatment options, but because of the potential for colonizing both inpatient and outpatient populations due to their ease of transmissibility, thus, creating a reservoir of bacterial resistance.

The data presented in this report include Enterobacteriaceae spp. that are resistant to carbapenems through the production of a carbapenemase. The first positive isolate from an inpatient identified as colonized or infected with CPE is eligible.

Subsequent positive isolates from the same patient in the same calendar year are eligible only if the patient tests positive for a different carbapenemase. If the patient was initially colonized and subsequently develops an infection with the same gene, within the same calendar year, only the infection is eligible for inclusion in surveillance. Data from previous years included in this report have been adjusted to reflect this change in reporting. 


\section{Appendix B: List of supplemental tables}

Table S1.1: Cases and incidence rates of healthcare-associated and community-associated Clostridioides difficile infection by region and hospital type, Canada, 2015-2018

Table S1.2: Antimicrobial resistance of healthcare and community-associated Clostridioides difficile infection isolates, Canada, 2015-2018

Table S1.3: Number and proportion of common ribotypes of HA-CDI and CA-CDI cases, Canada, 2015-2018

Table S2.1: Cases and incidence rates of healthcare-associated and community-associated methicillin-resistant Staphylococcus aureus bloodstream infections by region and hospital type, 2014-2018

Table S2.2: Number and proportion of select methicillin-resistant Staphylococcus aureus strain types identified

Table S3.1: Number of vancomycin-resistant Enterococci bloodstream infections incidence rates by region and hospital type, 2014-2018

Table S3.2: Number of healthcare-associated vancomycin-resistant Enterococci bloodstream infections and incidence rates, 2014-2018

Table S3.3: Number and proportion of vancomycin-resistant Enterococci bloodstream infections isolate types identified, 2014-2018

Table S3.4: Distribution of vancomycin-resistant Enterococci bloodstream (Enterococcus faecium) sequence type, 2014-2018

Table S4.1: Number of carbapenemase-producing Enterobacteriaceae infections and incidence rates by region, Canada, 2014-2018

Table S4.2: Number of carbapenemase-producing Enterobacteriaceae colonizations and incidence rates by region,

Canada, 2014-2018

Table S5: Number and proportion of main carbapenemase-producing pathogens identified 